\title{
Familiarity and difficulty of author-title materials for use in studies of long-term memory
}

\author{
RONALD H. HOPKINS \\ Washington State University, Pullman, Washington 99164
}

\begin{abstract}
Familiarity ratings were collected for 48 relatively well-known authors and for three representative titles by each author. Relative difficulty was determined by asking subjects to write an author's surname, given the set of three titles; independent groups were tested for unaided recall, for recall cued by the author's first and middle name, or for recognition. Correlations indicated that mean familiarity does predict difficulty scores. Overall recall levels were low, but were markedly improved by cuing or recognition testing. Thus, these materials should be useful in attempts to experimentally enhance retrieval from long-term memory.
\end{abstract}

Over the past decade or so, memory researchers have shown a resurgence of interest in the processes and structure of very long-term memory. This interest in retrieval of memories that may be several years old necessitates the use of preexperimentally acquired materials such as questions of general information, common knowledge, etc. A significant problem in much of this research is the lack of appropriate criteria for selection and counterbalancing of test items. The research reported here was undertaken to provide normative information about relative familiarity and difficulty. of materials to be used in the investigation of retrieval of proper names from long-term memory.

In particular, a set of relatively well-known author names and corresponding titles was selected from among those used in some earlier memory research (Hopkins \& Atkinson, 1968a, 1968b). The author names and titles were each rated on a scale of familiarity, and item difficulty was directly assessed in tests of recall and recognition of author surnames, given titles as stimuli.

\section{METHOD}

\section{Materials}

The original pool of author-title combinations, those used by Hopkins and Atkinson (1968a, 1968b), consisted of 156 authors of books, plays, essays, and short stories, along with three titles of representative works by each author. The materials had been gathered primarily from two encyclopedic sources (Hart, 1965; Hornstein \& Percy, 1956). Many authors were eliminated from the original pool for one or more of the following reasons: (1) To increase the likelihood that subjects had, in fact, stored information about an author, those writers were omitted who were infrequently or never remembered by a sample of students and staff at Stanford University (Hopkins \& Atkinson, 1968a, 1968b) or by students tested in pilot work at Washington State University. (2) To increase the homogeneity of the pool, writers associated primarily with nonfiction were

Requests for reprints or the table of familiarity ratings and difficulty scores should be sent to Ronald H. Hopkins, Department of Psychology, Washington State University, Pullman, Washington 99164. eliminated. (3) To avoid relatively short-term popularity effects and the need for continual updating of representative titles, authors were eliminated if a recent dictionary (American Heritage, Dictionary of the English Language, 1973) indicated they were still living. Thus, the resulting pool consisted of 48 relatively well-known, deceased authors of fiction with three titles by each author.

\section{Subjects}

The research participants were 230 introductory psychology students at Washington State University, participating in partial fulfillment of course requirements. None of the subjects had previously participated in long-term memory research.

\section{Procedure}

All subjects were tested in squads of 8 to 15 . Familiarity ratings were collected in one experiment and difficulty scores in another. Within each experiment, squads of subjects were randomly assigned, as they reported for testing, to one of the procedures described below.

Familiarity ratings. A total of 42 subjects rated author familiarity. Each subject was given a single sheet listing 48 author names in a random order. A 7-point rating scale with labels "very familiar" and "very unfamiliar" at the end points was printed at the top of the page. The instructions regarding the use of the rating scale were: "For example, if an author is very familiar to you, you could probably recall that author's name when given a title of one of his or her major works. For authors who are that familiar, you should place a 7 in the balnk preceding their names. At the opposite extreme, if you never heard of an author and knew nothing about him or her, you would put a 1 in the blank. Many of the authors will, of couse, be of intermediate familiarity. In every case simply rate an author's familiarity by assigning a number from 1 to 7 , using the scale at the top of the page."

Each of 38 subjects was given a response booklet containing a list of 144 titles ( 3 titles from each of the 48 authors) and asked to rate the familiarity of each title. In preparing the booklets, 36 titles were randomly assigned to each page, and the four different pages were scrambled before assembling each response booklet. The 7-point rating scale described above was duplicated at the top of each page. The instructions regarding the rating scale were similar to those for the author ratings: "For example, if you can recall the author of some title and may have read the book or know a good deal about it, you should place a 7 in the blank preceding the title, indicating that you are very familiar with that title. At the opposite extreme, if you have never heard of a title and know nothing about it, it would be very unfamiliar to you and you would assign a rating of 1. Many of the titles 
will, of course, be of intermediate familiarity. In every case, simply rate your familiarity with a title by assigning a number from 1 to 7, using the scale at the top of the page."

All subjects were instructed that they need not be concerned about the distribution of their ratings. The rating task was unpaced and all subjects finished in about $15 \mathrm{~min}$.

Item difficulty. The 48 title sets (each set consisting of three titles by a single author) were randomly assigned to the four pages of a response booklet, 12 sets per page, and the pages independently scrambled for each subject. The order of the three titles within each set was randomly determined. Subjects were instructed to write the surname for the author of each set of titles in the blank preceding the set, guessing if they wished. Fifty subjects were tested in each of three memory conditions: (1) unaided recall, in which no information about the correct author names was provided other than titles; (2) cued recall, in which the response blank for each set of titles was preceded by the correct author's first and middle names (or initials where appropriate, such as for F. Scott Fitzgerald); and (3) recognition, in which subjects were given an additional sheet containing an alphabetical listing of the full names of the 48 authors. The memory task was unpaced but all subjects were finished within $20 \mathrm{~min}$.

\section{Measures}

The mean familiarity rating for each author is referred to as AFAM. To facilitate discussion of the ratings, the three titles by each author were ranked on mean familiarity. The mean rating for an author's most familiar title is referred to as T1FAM (for Title 1 familiarity); similarly, the mean rating for an author's title of intermediate familiarity is referred to as T2FAM and the mean rating for an author's least familiar title as T3FAM.

The difficulty index derived from each of the memory conditions was simply the number of subjects who correctly named an author (maximum $=50$ ). For simplicity of presentation, the unaided recall score of each author is referred to as RECALL, the cued-recall score as CUREC, and the recognition score as RECOG.

\section{RESULTS}

The principal results are means and standard deviations of the familiarity ratings for each author and each title, and the difficulty scores for each author. The table of these data is much too lengthy for presentation here, and may be obtained from the author.

The reliability of AFAM scores was assessed by randomly splitting the subjects into two equal-sized groups and correlating the mean ratings of these subgroups. The resultant correlation coefficient was .93 . In addition, each subject's ratings were correlated with the mean ratings for all subjects. The mean of the latter correlations for AFAM was .65, indicating that subjects were reasonably consistent in using the rating scale. Similarly, the correlations between mean ratings of title familiarity for two subgroups was .96 , and the average correlation between individual ratings and group means for title familiarity was .73

Reliabilities for RECALL, CUREC, and RECOG were also computed by correlating the scores for two random, equal-sized subgroups from each condition. The resultant correlation coefficients were $.82, .96$, and .92 for RECALL, CUREC, and RECOG, respectively. Furthermore, RECALL scores were also available from 58 pilot subjects, and they correlated .97 with the RECALL scores discussed here. Thus, familiarity ratings and item difficulties were apparently both reliably measured.

The overall mean and standard deviation for each variable is presented in Table 1 . The summary data of most interest from the table are those for RECALL, CUREC, and RECOG. It is obvious that these materials are very difficult. For example, the mean RECALL score indicates that slightly less than $10 \%$ of the subjects ( 4.75 out of 50 ) were able, on the average, to provide a particular author name in unaided recall. However, the mean RECOG score is higher than the mean RECALL score by a factor of more than two to one $[t(47)=7.16$, $\mathrm{p}<.01]$, and CUREC scores are higher than RECALL scores by a factor of more than three to one $[t(47)=$ $7.18, p<.01]$. The apparent superiority of CUREC to RECOG scores [ $\mathrm{t}(47)=2.65, \mathrm{p}<.05$ ] is also interesting. Examination of the difficulty scores for each author, however, shows that the direction and magnitude of the difference between CUREC and RECOG varies considerably from author to author. Thus, the first name can be a very effective cue for retrieval of the surname, but the effect undoubtedly depends on such other factors as the uniqueness of the first name.

The matrix of correlations among the measures is presented in Table 2. Considering first the relations among the familiarity ratings in the left portion of the matrix, the coefficients suggest moderately strong relationships. Nevertheless, it does appear that author familiarity and title familiarity could be independently manipulated by appropriate selection of materials.

The correlations among the measures of memory difficulty are shown in the lower right portion of the matrix. Obviously, these correlations are substantial, even though the memory tasks were very different in overall difficulty.

The correlations between the indices of difficulty and mean familiarity ratings, in the upper right portion of Table 2, provide some evidence for the validity of the familiarity ratings. The relationship between famil-

Table 1

Overall Means and Standard Deviations for Mean Familiarity Ratings and for Difficulty Scores

\begin{tabular}{lccccccc}
\hline & AFAM & T1FAM & T2FAM & T3FAM & RECALL & CUREC & RECOG \\
\hline Mean & 3.82 & 4.06 & 2.34 & 1.58 & 4.75 & 15.17 & 11.40 \\
SD & 1.49 & 1.57 & 1.26 & .71 & 5.94 & 13.45 & 10.89 \\
\hline
\end{tabular}


Table 2

Simple Correlations Among Familiarity Ratings and Difficulty Scores

\begin{tabular}{lcccccc}
\hline & T1FAM & T2FAM & T3FAM & RECALL & CUREC & RECOG \\
\hline AFAM & .45 & .46 & .49 & .71 & .85 & .70 \\
T1FAM & & .56 & .31 & .59 & .45 & .70 \\
T2FAM & & & .61 & .42 & .36 & .48 \\
T3FAM & & & .49 & .43 & .72 & .87 \\
RECALL & & & & & .69 \\
CUREC & & & & & \\
\hline
\end{tabular}

iarity and difficulty scores appears to be a strong one. In fact, the multiple correlation of AFAM, T1FAM, T2FAM, and T3FAM with RECALL was .79. The corresponding correlation was even slightly higher for CUREC and RECOG, being .86 and .84, respectively. On the other hand, T2FAM and T3FAM appear to contribute little to the prediction of difficulty; the multiple correlation of AFAM and T1FAM with RECALL, CUREC, and RECOG was .77, .86, and .82, respectively. Thus, the multiple correlation is reduced very little by deleting T2FAM and T3FAM from among the predictors.

This latter conclusion is further substantiated by the third-order partial correlations. The partial correlation of each familiarity variable with each difficulty index, controlling for the remaining three familiarity variables, was computed with the results shown in Table 3 . It may clearly be seen that AFAM and T1FAM should be of the most concern in selecting materials for memory tasks comparable to those used here for indexing difficulty. However, the contribution of the different familiarity variables does seem to be task dependent.

\section{DISCUSSION}

Clearly, this is a small set of materials and may, therefore, be unsuitable for many kinds of long-term memory experiments. Furthermore, it is a difficult or unfamiliar set for many college students. On the other hand, the difference between RECALL and either CUREC or RECOG scores is substantial, and suggests that subjects in the sampled population knew much more about
Table 3

Partial Correlation Between Each Familiarity Variable and Each Difficulty Variable, With Other Familiarity Variables Controlled

\begin{tabular}{lccc}
\hline $\begin{array}{c}\text { Familiarity } \\
\text { Variable }\end{array}$ & RECALL & CUREC & RECOG \\
\hline AFAM & .56 & .80 & .54 \\
T1FAM & .43 & .19 & .59 \\
T2FAM & -.15 & -.16 & -.16 \\
T3FAM & .24 & .08 & .28 \\
\hline
\end{tabular}

the authors of various titles than could be recalled without assistance. Therefore, these materials may be very useful to researchers studying long-term memory by providing various retrieval cues or otherwise attempting to enhance retrieval.

\section{REFERENCES}

American heritage dictionary of the English language. New York: American Heritage, 1973.

HART, J. D. The Oxford companion to American literature (4th ed.). New York: Oxford University Press, 1965.

Hopkins, R. H., \& Atkinson, R. C. Degree of priming in the retrieval of authors' names from long-term memory. Psychonomic Science, 1968, 12, 399-400. (a)

Hopkins, R. H., \& Atrinson, R. C. First-letter clues in the retrieval of proper names from long-term memory. Psychological Reports, 1968, 23, 851-866. (b)

Hornstein, L. H., \& Percy, G. D. The reader's companion to world literature. New York: New American Library, 1956.

(Received for publication April 12, 1978.) 